\title{
HARD X-RAY OBSERVATION OF ABELL 496
}

\author{
Azita Valinia ${ }^{1,2}$, Keith Arnaud ${ }^{1,2}$, Michael Loewenstein ${ }^{1,2}$, Richard F. Mushotzky ${ }^{1}$, and \\ Richard Kelley ${ }^{1}$
}

Accepted for Publication in the Astrophysical Journal

\begin{abstract}
We report the results of hard X-ray observations of Abell 496 (A496), a nearby relaxed cluster, using the Rossi X-ray Timing Explorer (RXTE). The 3-20 keV spectrum of this cluster is well-modeled by a thermal component of $k T \sim 4.1 \mathrm{keV}$ plus a cooling flow with mass accretion rate of $\dot{M} \sim 285 \mathrm{M}_{\odot} \mathrm{yr}^{-1}$. The spectrum is equally well-modeled by a multi-temperature plasma component with a Gaussian temperature distribution of mean temperature $3.8 \mathrm{keV}$ and $\sigma_{k T} \sim 0.9 \mathrm{keV}$. The metallicity is found to be approximately $1 / 3$ solar; however, the $\mathrm{Ni} / \mathrm{Fe}$ ratio is about 3.6. No significant nonthermal emission at hard X-rays was detected for this cluster. We discuss the implications of the models presented here and compare them with the temperature profiles derived for this cluster using the Advanced Satellite for Cosmology and Astrophysics (ASCA). Our results are inconsistent with declining temperature profiles.
\end{abstract}

Subject headings: galaxies: clusters: individual (Abell 496) — X-rays: clusters

\footnotetext{
${ }^{1}$ Laboratory for High Energy Astrophysics, Code 662, NASA's Goddard Space Flight Center, Greenbelt, MD 20771; valinia@milkyway.gsfc.nasa.gov

${ }^{2}$ Department of Astronomy, University of Maryland, College Park, MD 20742
} 


\section{INTRODUCTION}

Observations over the last few years using ASCA and BeppoSAX have revolutionized our understanding of the X-ray emission from clusters of galaxies. Spatially-resolved spectroscopy has led to measurements of temperature and abundance variations in the hot gas trapped in the cluster gravitational wells. These measurements can be used to derive the total mass of clusters, to look for the signatures of merger events, and to follow the chemical evolution of the gas and the history of star formation in the cluster.

However, these results have not been without their controversies. Markevitch et al. (1998; 1999) have claimed, using ASCA observations, that most clusters have temperatures decreasing with distance from the cluster center. This analysis has been disputed using the same ASCA data by White (2000), using ROSAT PSPC data by Irwin, Bregman \& Evrard (1999), and using BeppoSAX data by Irwin \& Bregman (2000). Determining which of these analyses is correct is important for determining the true gravitational mass profiles in clusters of galaxies and for comparison with cosmological simulations.

In addition, there is a lively debate about the significance of any extended non-thermal emission from clusters of galaxies. This is claimed to be detected both at low energies (e.g. Lieu et al. 1996; Kaastra et al. 1999) and at high energies (e.g Fusco-Femiano et al. 1999; Rephaeli, Gruber \& Blanco 1999; Kaastra et al. 1999; Kaastra 2000). Such non-thermal emission could be due to inverse-Compton emission from cosmic microwave background photons scattering off relativistic electrons. If these electrons are also detected in the radio then the magnetic field strength can be determined. Alternatively, the emission could be due to non-thermal bremsstrahlung from suprathermal electrons accelerated in shocks or turbulence. However, Arabadjis \& Bregman (1999) found no evidence for an extremely soft non-thermal X-ray emission in a sample of clusters of galaxies.

Finally, there is a dispute about whether the $\mathrm{Fe} \mathrm{K} \alpha$ resonance line is optically thick to resonance scattering in the cores of clusters. If it were, this would mean that Fe abundances have been systematically underestimated. The detection of resonance scattering hinges on comparing the strengths of the Fe $\mathrm{K} \alpha$ and $\mathrm{Fe} \mathrm{K} \beta$ complexes. Since Fe K $\beta$ is confused with Ni $\mathrm{K} \alpha$ using current spectrometers the disagreement comes down to the Ni abundance assumed (Akimoto et al. 1997; Molendi et al. 1998; Dupke \& Arnaud 2000). The Fe/Ni ratio is a sensitive indicator of the types of supernovae whose products have enriched the gas (Dupke \& White 2000). Our understanding of the chemical evolution of clusters depends on this issue being resolved.

We will not answer all these questions with an RXTE observation of a single cluster. However, we will argue in this paper that high signal to noise $(\mathrm{S} / \mathrm{N})$ spectra from a large 
beam but well-calibrated detector such as the PCA can impose important constraints on models for the cluster emission. Previous high $\mathrm{S} / \mathrm{N}$ single beam observations of selected clusters have been done by Johnstone et al. (1992) using Ginga and by Henriksen \& White (1996) using HEAO-1. Since RXTE has no spatial resolution within the cluster it cannot be used to determine the temperature variation with position but it can test models proposed on the basis of observations with other satellites. The bandpass of the PCA extends above $20 \mathrm{keV}$, making it sensitive to non-thermal emission, particularly when observing cooler clusters. Finally, an observation of the entire cluster means that any effect due to (isotropic) resonance scattering will be absent.

\section{THE CLUSTER A496}

We chose to observe A496, a nearby $(z \approx 0.033)$ cluster with a moderate cooling flow. Recent optical and X-ray studies of this cluster (e.g. Markevitch et al. 1999; Durret et al. 2000) indicate that it has a regular morphology and a small amount of substructure. The analysis of the position, luminosity and velocity dispersion of the resident galaxies indicate that it is in a relaxed state with no strong environmental effects. The study of this cluster is, therefore, ideal as a prototype for understanding the properties of galaxy clusters. In addition, its relatively low X-ray temperature of 4-5 keV makes it an excellent prospect for testing for the presence of a high energy non-thermal tail to the spectrum.

Early observations of A496 with the Einstein SSS and Ginga LAC imply that metal abundances are centrally enhanced (White et al. 1994). Mushotzky et al. (1996) determined elemental abundances of $\mathrm{O}, \mathrm{Ne}, \mathrm{Mg}, \mathrm{Si}, \mathrm{S}, \mathrm{Ca}, \mathrm{Ar}$, and Fe from ASCA data and concluded that the observed ratio of the relative abundances of elements outside of the central cooling flow region is consistent with an origin of all the metals in Type II Supernovae. Dupke \& White (2000) confirm central iron abundance enhancements but also find a central nickel-toiron abundance ratio of approximately 4 that is consistent with the "convective deflagration" model in SN Ia explosion models.

Markevitch et al. (1999) derive maps and radial profiles of the gas temperature from ASCA measurements and find an average cluster temperature of $4.7 \pm 0.2 \mathrm{keV}$ (90\% error), about $10 \%$ higher than the wide beam single-temperature fits. Outside the central cooling flow region and within ASCA's field of view (fov), they describe the temperature profiles by a polytropic gas model with $\gamma \sim 1.24$. On the other hand, White (2000) finds the temperature outside of the cooling region to be isothermal. Dupke \& White (2000) also find a flat temperature profile outside the cooling radius. Kaastra (2000) notes that BeppoSAX observations of A496 suggest a hard tail in the spectrum. Irwin \& Bregman (2000) find a 
temperature rising from just below $4 \mathrm{keV}$ in the center to approximately $5 \mathrm{keV}$ at a radius of 9 arcminutes.

\section{OBSERVATIONS}

A496 was observed with the PCA and HEXTE instruments on board RXTE during December of 1998 and January of 1999 for a total duration of 100 ks. The PCA (Jahoda et al. 1996) has a total collecting area of $6500 \mathrm{~cm}^{2}$, an energy range of $2-60 \mathrm{keV}$, and energy resolution of $\sim 18 \%$ at $6 \mathrm{keV}$. The collimator field of view is approximately circular $\left(2^{\circ}\right.$ diameter) with FWHM of $1^{\circ}$. The HEXTE (Rothschild et al. 1998) consists of two clusters, each having a collecting area of $800 \mathrm{~cm}^{2}$, an energy range of $15-250 \mathrm{keV}$, energy resolution of $15 \%$ at $60 \mathrm{keV}$, and a field of view of $1^{\circ}$ FWHM. Furthermore, each cluster "rocks" along mutually orthogonal directions to provide background measurements away from the source.

We used the data taken when all 5 detectors of the PCA were on and the elevation angle from the limb of the Earth was greater than $10^{\circ}$. For this time duration, the background subtracted count rate over the $3-20 \mathrm{keV}$ band was $24.4 \pm 0.0279$ counts s$^{-1}$ for a total of more than 2.4 million counts. The total on-source integration time for the HEXTE detectors was $\sim 30 \mathrm{ks}$. The background subtracted count rate over the $15-30 \mathrm{keV}$ energy band was $0.00534 \pm 0.0313$ counts s$^{-1}$ and $-0.0259 \pm 0.0259$ counts $^{-1}$ for HEXTE clusters A and B, respectively. Hence, there was no positive detection with HEXTE. In what follows, we present results of our analysis of the PCA data.

\section{RESULTS}

\subsection{Reliability of the PCA calibration}

Our conclusions depend critically on the accuracy of the PCA response matrix used to fit the A496 data. Because the statistical accuracy of our data is so high we have to be particularly careful with systematic effects. We have analyzed observations of the Crab nebula obtained on December 15 and 29 of 1998 and January 13 of 1999, the same months that A496 was observed. We used pcarsp v2.43 to make the PCA response. We fit all three observations simultaneously over the $3-15 \mathrm{keV}$ band. Figure 1 shows the ratio of the data to model for the best fit absorbed power-law, which has an average slope of 2.167 and an average normalization of 7.3 photons $\mathrm{keV}^{-1} \mathrm{~cm}^{2} \mathrm{~s}^{-1}$ at $1 \mathrm{keV}$. The best fit column density

was $1.7 \times 10^{21} \mathrm{~cm}^{-2}$. The remaining systematic errors are at the $1 \%$ level or smaller. In the following description of our analysis of A496 we show ratios of data to model and assume that 
any variations above those seen in the Crab represent real differences between the assumed model and the data.

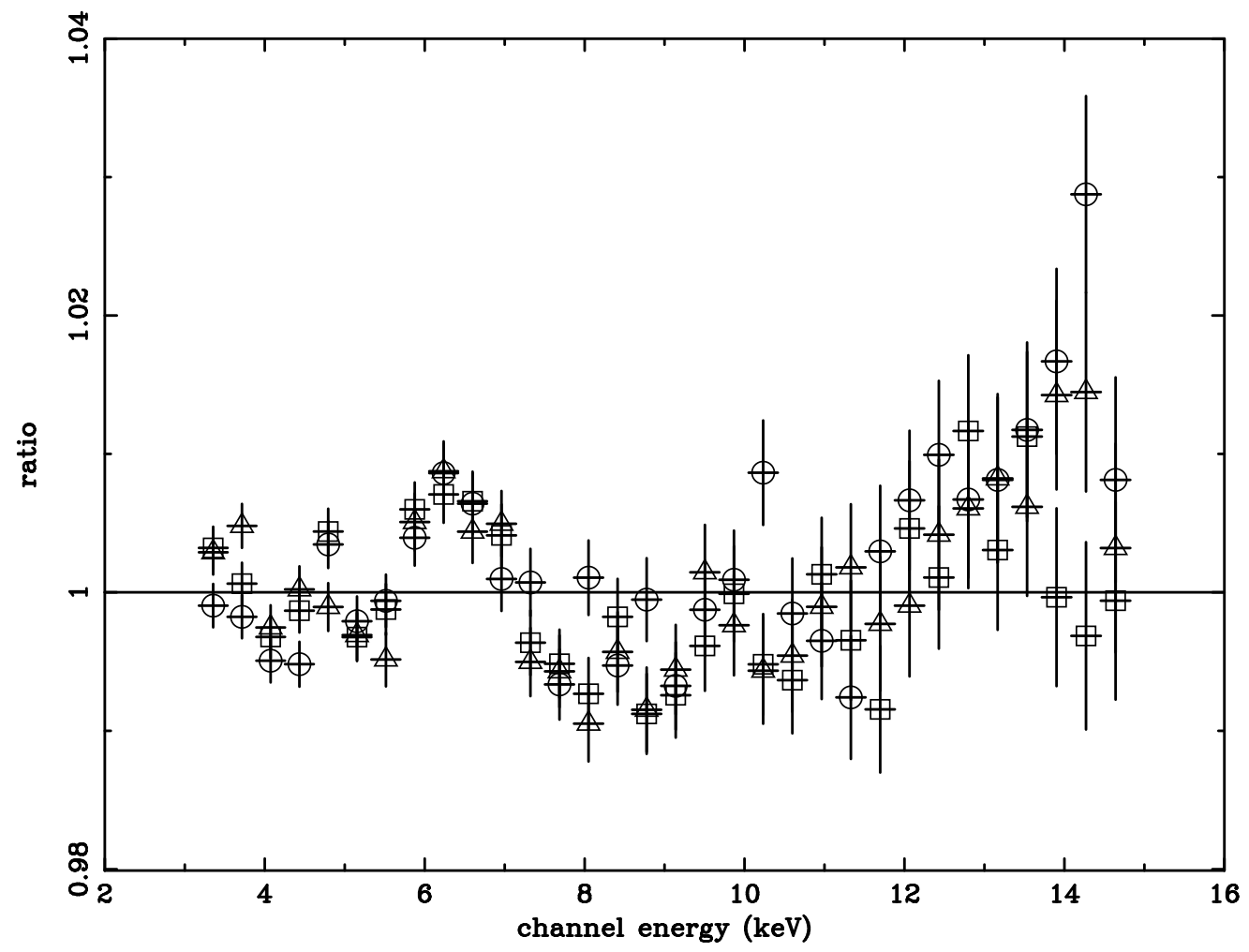

Fig. 1. - The ratio of the Crab nebula data to a best fit absorbed power law model, as measured by RXTE PCA during the same time interval that A496 was observed. Circle, square and triangle symbols correspond to data taken on $15 / 12 / 98,29 / 12 / 98$ and $13 / 1 / 99$, respectively.

\subsection{Thermal Models}

Table 1 shows all the models and their respective parameters used to fit the PCA data. For all the models reported, we fixed the Galactic hydrogen column density and the redshift at $4.5 \times 10^{20} \mathrm{~cm}^{-2}$ and 0.033 , respectively. We report errors (90\% confidence limit) for models with a $\chi^{2} / \nu$ of less than 2 .

Model A is a single isothermal mekal model (Mewe, Gronenschild, \& van den Oord 1985; Mewe, Lemen, \& van den Oord 1986; Liedahl, Osterheld, \& Goldstein 1995). The abundances of all elements are tied together and are allowed to vary, except for that of He which is fixed at the Solar value. However, this model is not satisfactory based on the $\chi^{2}$ statistic and the ratio of data to model shown in Figure 2a. Allowing the Ni abundance to 
vary independently improves the quality of the fit. Model B is therefore identical to model A except that we allowed the $\mathrm{Ni}$ abundance to vary independently. This model is a significant improvement over model A (Figure $2 \mathrm{~b}$ ). The best fit $\mathrm{Ni} / \mathrm{Fe}$ ratio in this model is $\sim 3.6$, consistent with the central Ni/Fe measured by Dupke \& White (2000) using ASCA. In all other models reported below, we fitted the data with both fixed and variable $\mathrm{Ni} / \mathrm{Fe}$ ratio. Since we consistently find that models with independently varying $\mathrm{Ni}$ abundance (specifically, with $\mathrm{Ni} / \mathrm{Fe}$ greater than one) produce the best fits, we only discuss models with independent $\mathrm{Ni}$ abundances hereafter.

Model B can be further improved. In particular, the cooling flow in A496 has been ignored. We construct model $\mathrm{C}$ by adding a cooling flow component to model $\mathrm{B}$. We tie the upper temperature of the cooling flow model to the temperature of the mekal component and also tie the abundances of the two components together. Of the models considered thus far, model $\mathrm{C}$ produces the best fit to the single beam PCA data (Figure 2c). The total unabsorbed $2-10 \mathrm{keV}$ flux in this model is $7.1 \times 10^{-11} \mathrm{erg} \mathrm{cm}^{-2} \mathrm{~s}^{-1}$. About $24 \%$ of the emission in this band is from the cooling flow component. From examination of archival A496 Ginga data, we find a total flux of $7.3 \times 10^{-11} \mathrm{erg} \mathrm{cm}^{-2} \mathrm{~s}^{-1}$ for this model. Hence, the RXTE and Ginga calibrations are in relatively good agreement.

Model D consists of two mekal components with their abundances tied together. Although, statistically, this model provides a good fit to the data, neither of the temperature components can be well constrained. Therefore, we have not reported any parameter errors for this model.

Model E is composed of a multi-temperature mekal component with a Gaussian temperature distribution. This model gives the best fit to the data (Figures $2 \mathrm{~d}$ and 3 ). The total unabsorbed 2-10 keV flux for this model is $7.0 \times 10^{-11} \mathrm{erg} \mathrm{cm}^{-2} \mathrm{~s}^{-1}$. The best fit $\sigma_{k T} \sim 0.9 \mathrm{keV}$ indicates that the temperature distribution is narrow. The variations in the ratio of data to model below $10 \mathrm{keV}$ are within the systematic errors observed for the Crab (i.e. compare Figures 1 and 2d).

Finally, we fit the data using a cooling flow plus the polytropic gas distribution derived by Markevitch et al. (1999) from ASCA data. The polytropic component was approximated by calculating the relative emission measures, derived using the Markevitch et al. (1999) $\beta$-model fits to the A496 surface brightness profile, in five temperature bins. Table 2 shows the emission measure fraction and emission-weighted temperature for each bin. Model $\mathrm{F}$ represents the temperature distribution for emission within the ASCA field of view (fov) only (Figure 2e). Model G extends the emission to the edge of the RXTE fov $\left(R \sim 2.4 h_{70}{ }^{-1}\right.$ $\mathrm{Mpc}$ ), holding the temperature constant at the value corresponding to the edge of the ASCA fov. Model $\mathrm{H}$ extrapolates the ASCA temperature profile using the polytropic index and 
density slope derived by Markevitch et al. The cooling flow component contributes $27 \%$, $34 \%$, and $36 \%$ to the total emission in models F, G, and $\mathrm{H}$, respectively. As seen from Table 1, none of these models fit the data well. Removing the cooling flow component from these models produces even poorer fits. Evidently, models with a broad temperature distribution that include substantial emission at relatively low temperatures are inconsistent with the data.

\subsection{Nonthermal Emission}

To search for evidence of hard X-ray nonthermal emission from A496, we added a power law component to the best fit model $\mathrm{E}$ in Table 1 . We fixed the photon index of the power law at 1.8. This is because a nonthermal hard X-ray power law with $\Gamma=1.8$ is detected in the BeppoSax hard X-ray spectrum of A2199 (Kaastra et al. 1999), a nearby $(z \approx 0.031$ ) cluster that is nearly identical in its X-ray properties to A496. Hence, we assume that the nonthermal component, if it exists, has similar properties to that in A2199. The $\chi^{2} / \nu$ for this model, however, decreases by 0.2 to 40.8/40 which represents a statistically insignificant improvement (see Table 1). The upper limit (90\% confidence limit) to the contribution of this component in the $3-20 \mathrm{keV}$ band is $6 \%$. The upper limit to the power law flux at $20 \mathrm{keV}$ is $1.1 \times 10^{-13} \mathrm{erg} \mathrm{cm}^{-2} \mathrm{~s}^{-1} \mathrm{keV}^{-1}$. The Cosmic X-ray background (CXB) fluctuations are about $8 \%$ rms of the mean CXB per RXTE fov (Valinia et al. 1999). The amplitude of the fluctuations amounts to $6.5 \times 10^{-14} \mathrm{erg} \mathrm{cm}^{-2} \mathrm{~s}^{-1} \mathrm{keV}^{-1}$ per RXTE fov at $20 \mathrm{keV}$. Therefore, the upper limit quoted above corresponds to a $2 \sigma$ fluctuation in the CXB.

For comparison we note that the non-thermal component in A2199 measured by Kaastra et al. (1999) using BeppoSAX has a flux at $20 \mathrm{keV}$ of $3.2 \times 10^{-13} \mathrm{erg} \mathrm{cm}^{-2} \mathrm{~s}^{-1} \mathrm{keV}^{-1}$. The contribution of the non-thermal component in the similar $3-20 \mathrm{keV}$ band is about $12 \%$. A comparable hard X-ray tail in A496 would have been easily detected by RXTE.

Neither allowing the power-law slope to be a free parameter, nor substituting Model C

for Model $\mathrm{E}$ as the thermal model, significantly improves the quality of the fit or increases the upper limit to the non-thermal contribution.

\section{DISCUSSION and CONCLUSIONS}

We have presented the results of our analysis of the highest signal-to-noise hard X-ray observations of A496. The large effective area and well calibrated response of RXTE enables us to place strong constraints on the distribution function of temperature in the total cluster 
volume. The distribution peaks at a temperature of $\approx 4.1 \mathrm{keV}$; however cooler emission is detected and can be modeled as an additional isothermal component or as a cooling flow $\left(\dot{M} \sim 285 \mathrm{M}_{\odot} \mathrm{yr}^{-1}\right)$. The data is also adequately modeled by a Gaussian distribution with $\sigma_{k T}=0.9 \mathrm{keV}$. Since a mass flow rate of $\sim 285 \mathrm{M}_{\odot} \mathrm{yr}^{-1}$ is high compared to Einstein, ROSAT and ASCA measurements $\left(\sim 100 \mathrm{M}_{\odot} \mathrm{yr}^{-1}\right.$, White et al. 1997; Peres et al. 1998; Allen et al. 2000) it is likely that the true model is a combination of a cooling flow and additional multi-temperature plasma. In principle, this can set limits on the degree of homogeneity of cluster gas (Allen et al. 1992). However, the temperature profile derived from ASCA data by Markevitch et al. (1999) produces a broad, flat distribution and is ruled out unless there is a temperature inversion outside the ASCA fov. However, no temperature inversion was found outside the half virial radius (roughly ASCA field of view in the case of A496) for clusters with $z>0.03$ in the Santa Barbara cluster simulation project (Frenk et al. 1999). These temperature gradients have been questioned (e.g., Irwin, Bregman, \& Evrard 1999; Kikuchi et al. 1999) and await XMM-NEWTON observations for confirmation.

Metal abundances of $1 / 3$ solar and a Ni/Fe ratio of 3.6 are found for this cluster. Both the peak in the temperature distribution and the metallicities are in excellent agreement with previous measurements of this cluster with ASCA (Mushotzky et al. 1996). The Ni/Fe ratio is consistent with that measured in the central region of the cluster (Dupke \& White 2000). If the apparently high $\mathrm{Ni} / \mathrm{Fe}$ ratio observed in the center of the cluster had been due to depressed emission from a resonantly-scattered Fe $\mathrm{K} \alpha$ line then a lower measured $\mathrm{Ni} / \mathrm{Fe}$ ratio would have been measured by RXTE since it collects all the Fe K $\alpha$ photons, whether or not they are scattered. So, we conclude that the measured Ni/Fe ratio is a true measurement of the relative abundances of these two elements in the central region and is not due to optical depth effects. Since Ni is mostly originated in type I SNe, the ratio of $\mathrm{Ni} / \mathrm{Fe}$ sets constraints on the production of Fe in type I SNe throughout the cluster.

No significant hard X-ray non-thermal emission was detected. Hard X-ray emission in clusters of galaxies is expected from detailed models of intracluster medium (ICM) evolution. The contributing processes are expected to be inverse Compton (IC) scattering, synchrotron radiation, nonthermal bremsstrahlung emission and Coulomb losses to the ICM (e.g. Sarazin 1999). Since radiation fields (e.g. optical/IR and X-ray) and magnetic fields $(\lesssim 1 \mu \mathrm{G})$ are low in the ICM, highly relativistic electrons mainly lose energy via IC scattering off cosmic microwave background photons (Sarazin \& Lieu 1998). For an electron of energy $20 \mathrm{keV}$, its lifetime is dominated by IC losses given by

$$
t_{I C}=\frac{\gamma m_{e} c^{2}}{\frac{4}{3} \sigma_{T} c \gamma^{2} U_{C M B}} \approx 4.8 \times 10^{8}\left(\frac{\gamma}{4800}\right)^{-1} \mathrm{yr},
$$

where $\gamma$, the Lorentz factor, is about 4800. Clearly, these particles have very short lifetimes and are present only in clusters where there has been current or very recent particle 
acceleration such as in ICM shocks or cluster mergers. In this context, the detection of nonthermal emission from A2199 with BeppoSax is surprising. A more plausible mechanism for producing hard tails in clusters without radio halos is non-thermal bremsstrahlung by suprathermal (but subrelativistic) electrons (Kempner \& Sarazin 2000, Blasi 2000). Ongoing turbulent particle acceleration is still required to overcome Coulomb losses; however, the power requirements may be acceptable - even for relatively relaxed clusters. A2199 has very similar properties to A496 - it is at a redshift of 0.032 with an average cluster temperature of $4.2 \mathrm{keV}$, and it is therefore somewhat surprising that there may be a hard tail in the former but not the latter. We caution that the existence of nonthermal hard X-ray emission from clusters of galaxies is not experimentally established as a general phenomenon. To date, positive detection of this component has been reported only for the Coma cluster (Rephaeli, Gruber, \& Blanco 1999; Fusco-Femiano et al. 1999), A2256 (Fusco-Femiano et al. 2000) and A2199. However, Coma and A2256 are very dynamically active clusters undergoing merging while A2199 is not. A complete hard X-ray survey of galaxy clusters is needed to establish whether the presence of this component is common or exceptional.

We thank Keith Jahoda for providing the Crab data. This research has taken advantage of the LEDAS archival database. 

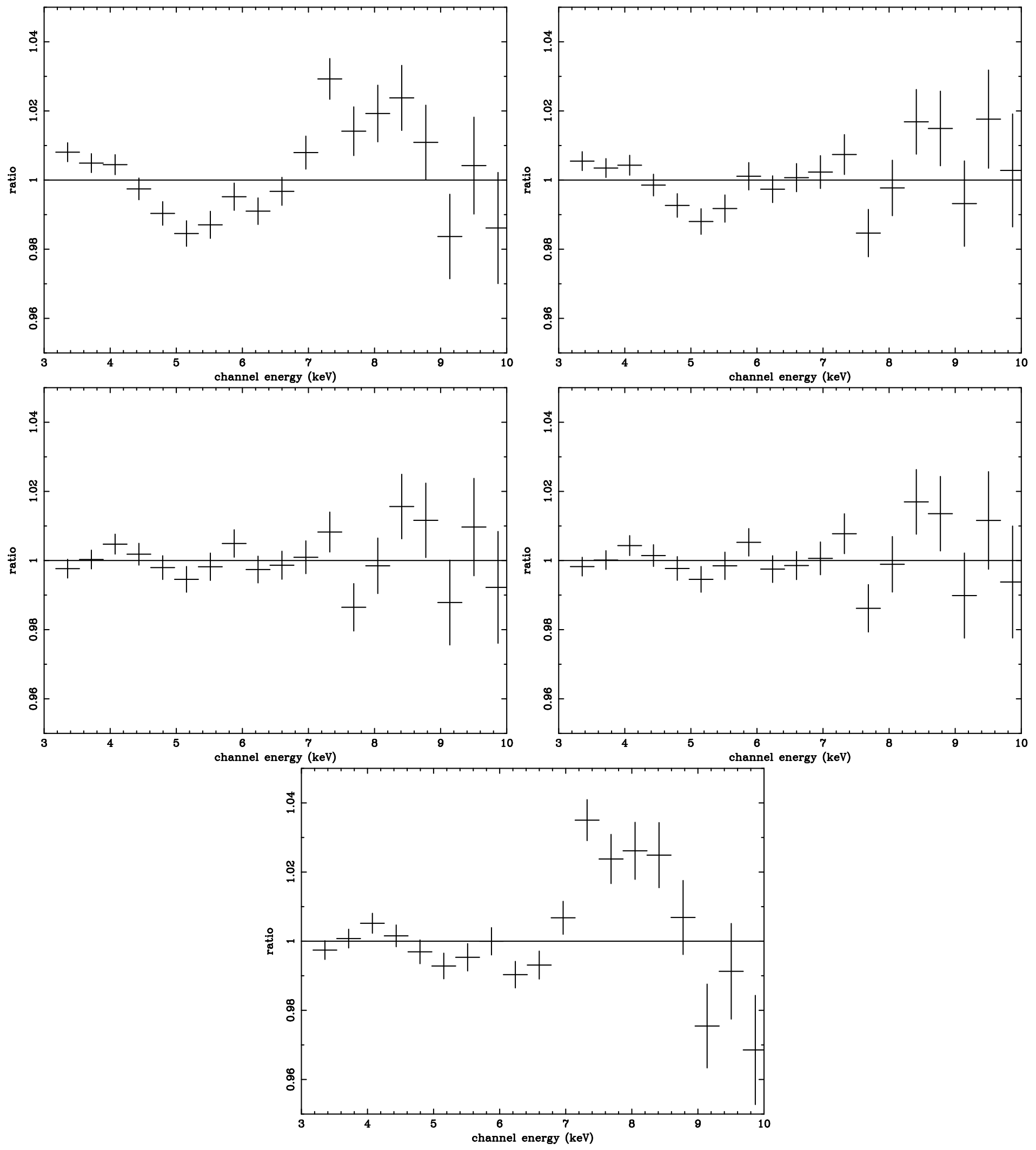

Fig. 2.- (From top left to right) The ratio of A496 data taken with RXTE PCA to model for model A (a), model B (b), model C (c), model E (d), and model F (e). 


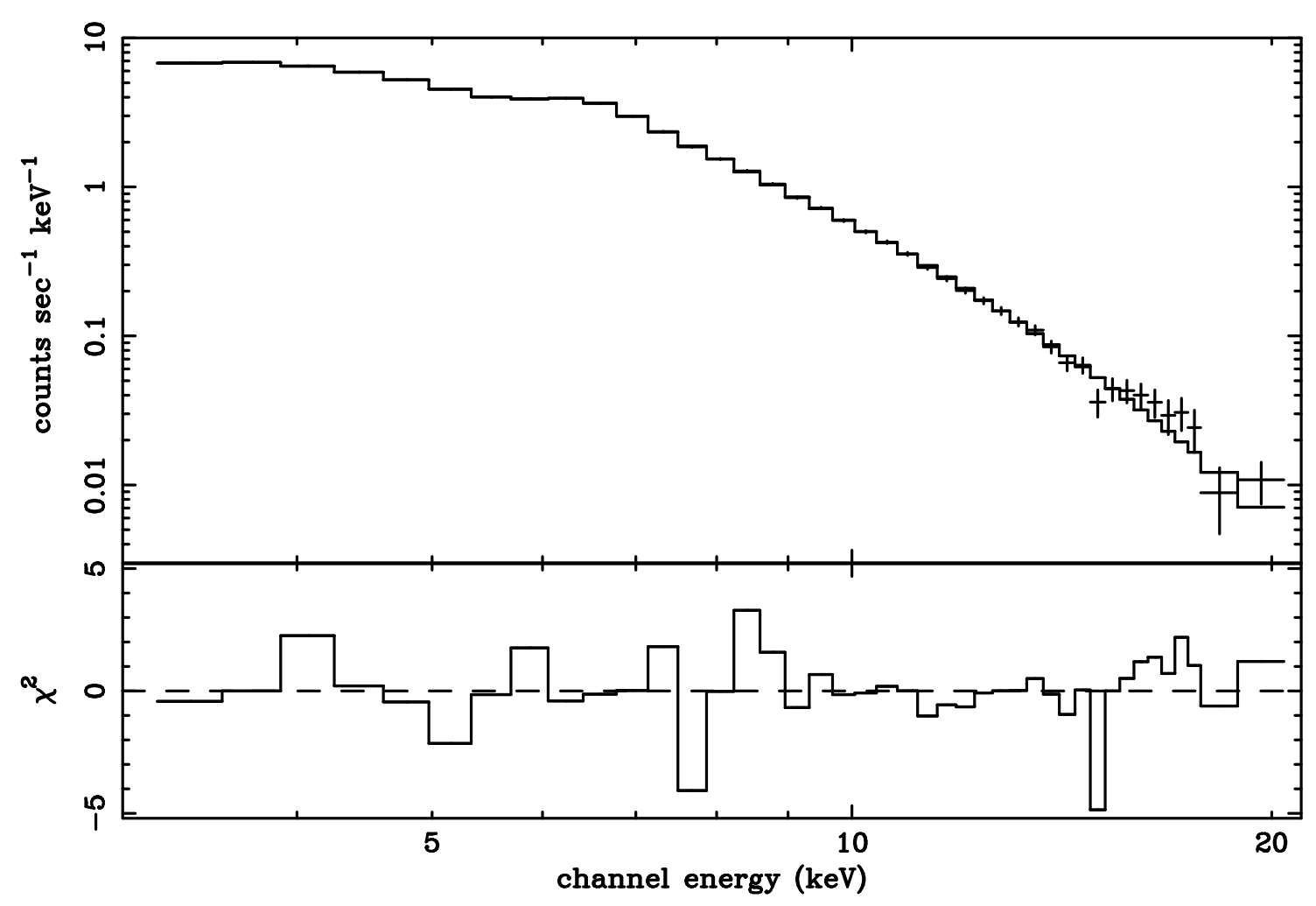

Fig. 3.- RXTE PCA data of A496 and folded model E in Table 1. $\chi^{2}$ is plotted in the lower panel. 


\section{REFERENCES}

Akimoto, F., Tawara, Y., Kumada, A., \& Yamashita, K., 1997, in X-ray imaging and spectroscopy of cosmic hot plasmas, ed. F. Makino, K. Mitsuda (Tokyo: University Academic Press)

Allen, S. W., Fabian, A. C., Johnstone, R. M., Arnaud, K. A., Nulsen, P. E. J., 2000, MNRAS, submitted

Allen, S. W., Fabian, A. C., Johnstone, R. M., Edge, A. C., \& Nulsen, P. E. J., 1992, 245, 51

Arabadjis, J. S., \& Bregman, J. N. 1999, ApJ, 514, 607

Blasi, P. 2000, ApJ, in press

Dupke, R. A., \& White, R. E., 2000, ApJ, 528, 139

Dupke, R. A., \& Arnaud, K. A., 2000, ApJ, submitted.

Durret, F., Adami, C., Gerbel, D., \& Pislar, V., 2000, astro-ph/0002519

Frenk, C. S., et al. 1999, ApJ, 525, 554

Fusco-Femiano, R., et al., 1999, ApJL, 513, L21

Fusco-Femiano, R., Dal Fiume, D., De Grandi, S., Feretti, L., Giovannini, G., Grandi, P., Malizia, A., Matt, G., \& Molendi, S., 2000, astro-ph/0003141

Henriksen, M. J., \& White, R. E., 1996, ApJ, 465, 515

Irwin, J. A., Bregman, J. N., \& Evrard, A. E. 1999, ApJ, 519, 518

Irwin, J. A., \& Bregman, J. N., 2000, ApJ, submitted, astro-ph/0003123

Johnstone, R. M., Fabian, A. C., Edge, A. C., \& Thomas, P. A., 1992, MNRAS, 255, 431

Jahoda, K., Swank, J. H., Giles, A. B., Stark, M. J., Strohmayer, T., Zhang, W., \& Morgan, E. H. 1996, Proc. SPIE, 2808, 59

Kaastra, J. S., et al., 1999, ApJL, 519, 119

Kaastra, J. S., 2000, Adv.Space.Res., 25, 741

Kempner, J. C., \& Sarazin, C. L. 2000, ApJ, in press 
Kikuchi, K., Furusho, T., Ezawa, H., Yamasaki, N. Y., Ohashi, T., Fukazawa, Y., \& Ikebe, Y. 1999, PASJ, 51, 301

Lieu, R., Mittaz, J. P. D., Bowyer, S., Lockman, F. J., Hwang, C-Y., \& Schmitt, J. H. M. M., 1996, ApJ, 458, L5

Markevitch, M., Forman, W. R., Sarazin, C. L., \& Vikhlinin, A., 1998, ApJ, 503, 77

Markevitch, M., Vikhlinin, A., Forman, W. R., \& Sarazin, C. L., 1999, ApJ, 527, 545

Mewe, R., Gronenschild, E. H. B. M., \& van den Oord, G. H. J. 1985, A\&AS, 62, 197

Mewe, R., Lemen, J. R., \& van den Oord, G. H. J., 1986, A\&AS, 65, 511

Molendi, S., Matt, G., Antonelli, L. A., Fiore, F., Fusco-Femiano, R., Kaastra, J., Maccarone, C., Perola, C., 1998, ApJ, 499, 608

Mushotzky, R., et al., 1996, ApJ, 466, 686

Peres, C. B., Fabian, A. C., Edge, A. C., Allen, S. W., Johnstone, R. M., White, D. A., 1998, MNRAS, 298, 416

Rephaeli, Y., Gruber, D., \& Blanco, P., 1999, ApJL, 511, L21

Rothschild, R. E., Blanco, P. R., Gruber, D. E., Heindl, W. A., MacDonald, D. R., Marsden, D. C., Pelling, M. R., Waune, L., R., \& Hink, P. L. 1998, ApJ, 496, 538

Sarazin, C. L., 1999, ApJ, 520, 529

Sarazin, C. L., \& Lieu, R. 1998, ApJ, 494, L177

Valinia, A., Henriksen, M. J., Loewenstein, M., Roettiger, K., Mushotzky, R. F., \& Madejski, G., 1999, ApJ, 515, 42

White, D. A., Jones, C., \& Forman W., 1997, MNRAS, 292, 419

White, D. A., 2000, MNRAS, 312, 663

White, R. E., Day, C. S. R., Hatsukade, I., \& Hughes, J. P., 1994, ApJ, 433, 583 
Table 1. Models and Best Fit Parameters

\begin{tabular}{cccccccc}
\hline \hline Model & $k T(\mathrm{keV})$ & $\sigma_{k T}(\mathrm{keV})$ & $\begin{array}{c}\text { Abundances } \\
(\times \text { solar })\end{array}$ & $\mathrm{Ni}$ & $\mathrm{Ni} / \mathrm{Fe}$ & $\dot{M}\left(\mathrm{M}_{\odot} \mathrm{yr}^{-1}\right)$ & $\chi^{2} / \nu^{*}$ \\
\hline $\mathrm{A}$ & 3.99 & - & 0.32 & 0.32 & 1 & - & $129.5 / 43$ \\
$\mathrm{~B}$ & $3.93 \pm 0.02$ & - & $0.32 \pm 0.01$ & $1.17 \pm 0.18$ & 3.7 & - & $69.7 / 42$ \\
$\mathrm{C}$ & $4.14_{-0.08}^{+0.10}$ & - & $0.33 \pm 0.01$ & $1.15 \pm 0.19$ & 3.5 & $285 \pm 88$ & $41.7 / 40$ \\
$\mathrm{D}$ & $3.5 \& 6.8$ & - & 0.34 & 1.26 & 3.7 & - & $41.0 / 39$ \\
$\mathrm{E}$ & $3.81_{-0.06}^{+0.05}$ & $0.90 \pm 0.14$ & $0.34 \pm 0.01$ & $1.21 \pm 0.19$ & 3.6 & - & $41.0 / 41$ \\
$\mathrm{~F}$ & - & - & 0.22 & 0.29 & 1.3 & 315 & $138.4 / 40$ \\
$\mathrm{G}$ & - & - & 0.27 & 0.33 & 1.2 & 270 & $292.5 / 40$ \\
$\mathrm{H}$ & - & - & 0.25 & 0.37 & 1.5 & 295 & $503.4 / 40$ \\
\hline
\end{tabular}

${ }^{*}$ chi-squared over degrees of freedom

Table 2. Emission Measure Percentages Assuming ASCA T-Profile*

\begin{tabular}{clll}
\hline \hline Temperature Bin (keV) & Model F & Model G & Model H \\
\hline $5.2-6.2$ & $18(5.6)$ & $10(5.6)$ & $10(5.6)$ \\
$4.2-5.2$ & $27(4.7)$ & $18(4.7)$ & $18(4.7)$ \\
$3.2-4.2$ & $42(3.7)$ & $22(3.7)$ & $22(3.7)$ \\
$2.2-3.2$ & $13(3.0)$ & $50(3.0)$ & $30(2.7)$ \\
$<2.2$ & 0 & 0 & $20(2.0)$ \\
\hline
\end{tabular}

*emission-averaged temperature $(\mathrm{keV})$ in each bin in parenthesis 